\title{
Onset of Angina Pectoris Event
}

National Cancer Institute

\section{Source}

National Cancer Institute. Onset of Angina Pectoris Event. NCI Thesaurus. Code C67096.

The beginning of the experience of the symptom complex of angina pectoris. 